\title{
Renal hemodynamics by return cannular position of extracorporeal membrane oxygenation in swine
}

\author{
Hee Jung Kim ${ }^{1}$, Seong Cheol Jeong ${ }^{2}$, Jae Seung Jung ${ }^{1}$, In Seup Kim², Choon-Hak Lim³ , Ho Sung Son ${ }^{1}$ \\ ${ }^{1}$ Department of Thoracic and Cardiovascular Surgery, Korea University Anam Hospital, College of Medicine, Korea University, Seoul, Korea; \\ ${ }^{2}$ Department of Thoracic and Cardiovascular Surgery, Uijeongbu St. Mary's Hospital, The Catholic University of Korea, College of Medicine, \\ Uijeongbu, Korea; ${ }^{3}$ Department of Anesthesiology and Pain Medicine, Korea University Anam Hospital, College of Medicine, Korea University, \\ Seoul, Korea \\ Contributions: (I) Conception and design: HJ Kim, HS Son; (II) Administrative support: HS Son; (III) Provision of study materials or patients: HJ \\ Kim, SC Jeong; (IV) Collection and assembly of data: HJ Kim, SC Jeong; (V) Data analysis and interpretation: All authors; (VI) Manuscript writing: \\ All authors; (VII) Final approval of manuscript: All authors. \\ Correspondence to: Ho Sung Son, MD, PhD. Department of Thoracic and Cardiovascular Surgery, Korea University Anam Hospital, College of \\ Medicine, Korea University, 73, Inchon-ro, Seongbuk-gu, Seoul 02841, Korea. Email: hssonmd@korea.ac.kr.
}

\begin{abstract}
Background: Whether arterial return cannula position affects the kidney during Veno-Arterial extracorporeal membrane oxygenation (ECMO) is unclear. Therefore, we compared hemodynamic parameters and acute kidney injury (AKI) biomarkers between ascending aorta return (aECMO) and femoral artery return ECMO (fECMO) in swine to evaluate the effect of cannula position on the kidney.

Methods: A total of twelve swines were allocated randomly into two groups. ECMO was maintained for 6h. Hemodynamic parameters including mean arterial pressure (MAP), renal arterial flow rate (AF), energy equivalent pressure (EEP), and surplus hemodynamic energy (SHE) were measured at the left renal artery. For evaluation of kidney injury, samples were obtained for blood urea nitrogen, creatinine, cystatin $\mathrm{C}$, and neutrophil gelatinase-associated lipocalin (before ECMO, and 1, 3, and $6 \mathrm{~h}$ after initiating ECMO).

Results: Before the start of ECMO, hemodynamic parameters were not different between the two groups. With regard to the rate of change before and after ECMO, the fECMO group showed a significantly higher increase in MAP, AF, and EEP and a greater decrease in SHE than the aECMO group $(\mathrm{P}<0.001)$. In intergroup analysis, no significant difference in time-dependent trends were observed for biochemical laboratory levels.
\end{abstract}

Conclusions: fECMO support was associated with a higher energy profile at the renal artery than that with aECMO, whereas pulsatility was decreased.

Keywords: Extracorporeal membrane oxygenation (ECMO); aorta; femoral artery; arterial pressure; acute kidney injury (AKI)

Submitted Jul 02, 2019. Accepted for publication Sep 09, 2019.

doi: $10.21037 /$ jtd.2019.09.73

View this article at: http://dx.doi.org/10.21037/jtd.2019.09.73

\section{Introduction}

Extracorporeal membrane oxygenation (ECMO) is widely used for treatment of cardiopulmonary failure. However, complications following ECMO are not uncommon, and the sequelae are serious in patients with critical illness. Because patients receiving ECMO likely have severe vital organ dysfunction and limited cardiopulmonary reserve, those with complications from ECMO show poor survival rates and higher risk of morbidity (1). The survival rate in patients receiving ECMO assist for cardiac dysfunction was only 40$90 \%$ (1-3). The most common complication during ECMO is acute renal impairment, with worse prognosis in patients with renal failure than in those with preserved renal function $(1,4,5)$. 
Therefore, protection or preservation of renal function is an essential concern in the ECMO management.

Unstable hemodynamic status and circulation are a major cause of renal impairment. In experimental observation, a decrease in renal perfusion to $<50 \%$ provoked impairment of energy metabolism and elevated lactic acid (6). Addition of ECMO or cardiopulmonary bypass to the systemic circulation can replace the deficit of renal perfusion in patients with decreased cardiac output. However, concerns regarding monitoring and management of cardiac assist device for optimal renal perfusion remain subjects of debate (7-9).

In circulatory support, return cannula position is an important issue for optimized organ perfusion (central $v s$. peripheral). The central approach is achieved by positioning of the return cannula (arterial cannula) near the heart (ascending aorta), while the peripheral approach is achieved by positioning of the return cannula at a distal artery (primarily the femoral artery). Many studies have assessed the differences in clinical outcomes between the two techniques in experimental and clinical settings (10-14). However, there is lack of data on renal preservation.

The aim of this study was to evaluate the impact of return cannula positioning on renal preservation using a central and peripheral approach for ECMO by measurement of changes in hemodynamic and biochemical parameters in swine.

\section{Methods}

\section{Animal study protocol and preparation}

This study was approved by Korea University Institutional Animal Care and Use Committee. All animals were treated according to the Guide for the Care and Use of Laboratory Animals issued by the Korea University School of Medicine. Healthy 40-60 kg female Yorkshire swine were used in this study. A total of 12 swine were randomly assigned to a central ECMO group (aECMO) or a peripheral ECMO group (fECMO). Those with aECMO was received ascending aorta return cannular (arterial cannula), while fECMO was received femoral artery return cannula. Swine were premedicated with intramuscular Zoletil $^{\circledR}$ (Virbac, France) $4.4 \mathrm{mg} / \mathrm{kg}$. Weight was measured, endotracheal tube insertion was performed, and general anesthesia was maintained with inhalation of isoflurane $1 \%$. Mechanical ventilation was set for a tidal volume of $10-15 \mathrm{~mL} / \mathrm{kg}$ and a respiratory rate of $20-25$ breaths $/ \mathrm{min}$. For muscle relaxation, Vecaron ${ }^{\circledR}$ (Reyon, Korea) was administrated before skin incision. An intravenous fluid

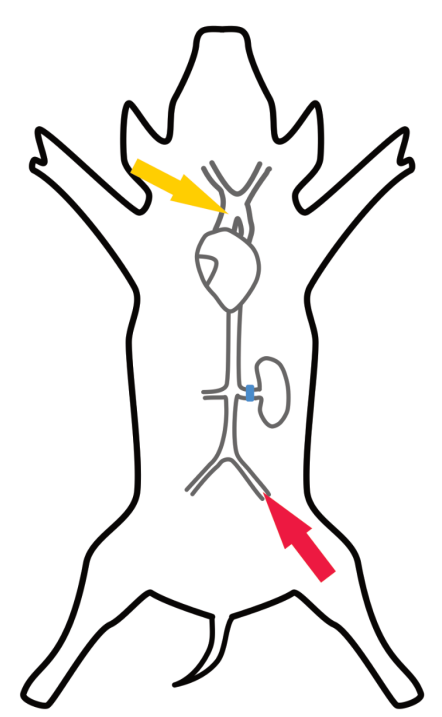

Figure 1 Experimental model for extracorporeal membrane oxygenation comparing between central and peripheral arterial return cannular. Central arterial return had ascending aorta cannula (yellow arrow) and peripheral arterial return had femoral artery cannula (red arrow). Renal artery flow and pressure were measured at the left renal artery (blue square).

route was established at the right external jugular vein. Median sternotomy and thoracoabdominal incision through the extraperitoneal space were performed in both groups. The ascending aorta, abdominal aorta, and left renal artery were dissected and exposed. To determine baseline cardiac output, arterial blood flow through the ascending aorta was measured using a 20-mm flowmeter (Transonic Systems, USA). For hemodynamic measurement, a 20-G angiocatheter was inserted into the renal artery to monitor renal artery pressure and a $3-\mathrm{mm}$ ultrasonic flow probe (Transonic systems, USA) was placed in the left renal artery for flow measurements. Blood pressure was measured using a sensor (PS9030VY; (Sensortechnics, Germany) and amplifier (ST-AM100; Senstech, Korea). Blood flow was measured using a perivascular flow probe (Transonic Systems, Inc., USA) and flowmeter module (T402; Transonic). Sampling was performed at 1,000 Hz/s. Data were collected (using NI USB 6009; National Instruments, Austin, TX, USA) and stored with a data acquisition program (Labview Software (National Instruments, USA). All hemodynamic parameters were measured under beating heart conditions (Figure 1).

ECMO was prepared using a centrifugal pump (Rotaflow; Maquet Cardiovascular, Germany) and hollow fiber 
oxygenator (Capiox SX10R; Terumo, Japan). Perfusate was made with Ringer's lactate solution and $20 \%$ albumin. Heparin was injected at $3 \mathrm{mg} / \mathrm{kg}$. The venous cannula was placed through the femoral vein $\left(17-\mathrm{F} \mathrm{DLP}^{\circledR}\right.$ femoral cannula; Medtronics, USA), with the cannula tip in the right atrium for venous drainage in both groups.

The arterial cannula was placed in the ascending aorta in the aECMO group (12-F DLP ${ }^{\circledR}$ Pediatric One-Piece arterial cannula, Medtronics), and in the femoral artery using Seldinger technique (14-F, $\mathrm{DLP}^{\circledR}$ femoral cannula, Medtronics) in the $\mathrm{EECMO}$ group.

After initiation of ECMO, the flow rate of ECMO was increased and maintained at premeasured ascending aorta flow rate (permitted range $\pm 10 \%$ ). Crystalloid fluid was administrated at $4 \mathrm{~mL} / \mathrm{kg} / \mathrm{h}$. Target mean blood pressure was $>50 \mathrm{mmHg}$. At the end of the experiment, all animals were sacrificed under anesthesia according to our institutional guidelines.

\section{Acquisition of bemodynamic and biochemical parameters}

$\mathrm{ECMO}$ was maintained for $6 \mathrm{~h}$ in each animal. Renal artery hemodynamic parameters including blood flow $(\mathrm{mL} / \mathrm{min})$ and blood pressure $(\mathrm{mmHg})$ at left renal artery were sequentially recorded before and after initiation of ECMO, and every $30 \mathrm{~min}$ thereafter. Energy equivalent pressure (EEP) was calculated using measured parameters and Shepard's energy formula (15-17).

\section{EEP}

Energy equivalent pressure represents total sum of energy.

Energy equivalent pressure $(m m H g)=(j p f d t) /(f f d t)$

$\mathrm{f}=$ arterial flow rate $(\mathrm{L} / \mathrm{min}), \mathrm{p}=$ arterial pressure $(\mathrm{mmHg})$, time $=$ single pulse cycle

\section{Surplus hemodynamic energy (SHE)}

SHE represents pulsatile energy.

Surplus bemodynamic energy $\left(\mathrm{erg} / \mathrm{cm}^{3}\right)=1,332 \times($ EEP mean arterial pressure)

Blood was collected before and after initiation of ECMO, and at 3 and $6 \mathrm{~h}$. Blood samples were used to quantify biochemical parameters (BUN, creatinine) and biomarkers [cystatin C, neutrophil gelatinase-associated lipocalin (NGAL)]. Enzyme-linked immunosorbent assay (ELISA) was performed with biomarker kits (Pig cystatin C ELISA kit; Cusabio, China and Pig neutrophil gelatinase-associated lipocalin ELISA kit; Cusabio).

\section{Statistical methods}

Categorical variables, presented as frequencies and percentages, were compared using the chi-square. Continuous variables, expressed as mean \pm standard deviation, were compared using Student's $t$-test.

Repeated-measures analysis of variance was used to evaluate the changes in BUN, creatinine, cystatin $\mathrm{C}$, and NGAL across time points between groups (before and after initiation of ECMO, and at 3 and $6 \mathrm{~h}$ ).

All reported $\mathrm{P}$ values were 2 -tailed, and $\mathrm{P}<0.05$ was considered statistically significant. Statistical analysis was performed with SPSS 20.0 (IBM, USA) and R 3.4.4 (R Foundation for Statistical Computing, Austria).

\section{Results}

Twelve swine were randomly assigned to aECMO and fECMO groups. Mean weight was was $39.6 \pm 6.8 \mathrm{~kg}$ in the aECMO group and $37.8 \pm 4.7 \mathrm{~kg}$ in the $\mathrm{EECMO}$ group $(\mathrm{P}=0.62)$ Before initiation of ECMO, arterial flow through the ascending aorta was $2.34 \pm 0.69 \mathrm{~L} / \mathrm{min}$ in the aECMO group and $2.54 \pm 0.49 \mathrm{~L} / \mathrm{min}$ in the $\mathrm{fECMO}$ group $(\mathrm{P}=0.6)$. After initiation of ECMO, the flow rate was maintained at $2.34 \pm 0.70 \mathrm{~L} / \mathrm{min}$ in the aECMO group and $2.56 \pm 0.49 \mathrm{~L} / \mathrm{min}$ in the $\mathrm{fECMO}$ group $(\mathrm{P}=0.55)$.

Before initiation of ECMO, baseline renal artery hemodynamic parameters including mean arterial pressure (MAP), arterial flow rate (AF), EEP, and SHE showed no significant differences between the 2 groups (MAP: $52.01 \pm 6.83$ vs. $50.59 \pm 6.51 \mathrm{mmHg}$; AF: $232.5 \pm 193.6$ vs. $138.0 \pm 51.6 \mathrm{~mL} / \mathrm{min}$; EEP: $55.52 \pm 2.66$ vs. $54.11 \pm 7.35 \mathrm{mmHg}$; SHE: $5,171.96 \pm 2,170.29$ vs. $4,646.39 \pm 3,217.30 \mathrm{erg} / \mathrm{cm}^{3}$, in the aECMO and $\mathrm{EECMO}$ groups, respectively; all $\mathrm{P}$ were no significant) (Table 1).

\section{Rate of change in hemodynamic parameters after initiation of ECMO}

After initiation of ECMO, the rate of change in hemodynamic parameters was determined. Compared with baseline values, both groups showed a significant increase in MAP, AF, and EEP after initiation of ECMO, but SHE was decreased $(\mathrm{P}<0.05)$ (Table 2, Figure 2). The fECMO group showed a greater increase in the rate of change for MAP, AF, and EEP than the aECMO group. However, the negative rate of change for SHE was lower in the $\mathrm{EECMO}$ group than in the aECMO group $(\mathrm{P}<0.05)($ Table 2, Figure 1). 
Table 1 Hemodynamic renal artery parameters before and after initiation of extracorporeal membrane oxygenation using central and peripheral approaches

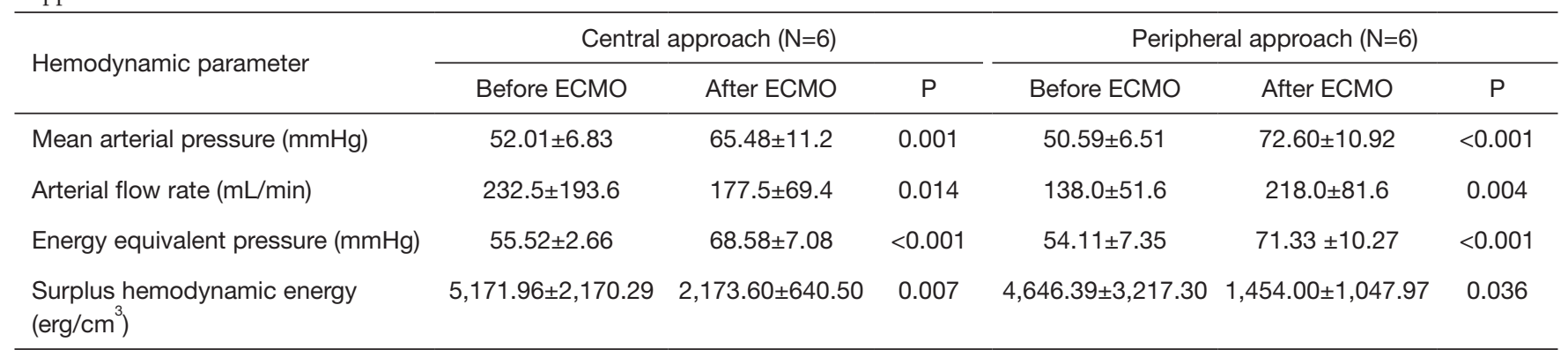

ECMO, extracorporeal membrane oxygenation.

Table 2 Comparison of hemodynamic change rates before and after initiation of extracorporeal membrane oxygenation using central and peripheral approaches

\begin{tabular}{lccc}
\hline Hemodynamic parameter & Central cannula $(\mathrm{N}=60)$ & Peripheral cannula (N=60) & $P$ value \\
\hline Mean arterial pressure (\%) & $29.24 \pm 17.1$ & $58.35 \pm 32.97$ & $<0.001$ \\
Arterial flow rate (\%) & $25.35 \pm 70.47$ & $79.45 \pm 48.48$ & $<0.001$ \\
Energy equivalent pressure (\%) & $29.47 \pm 15.89$ & $48.04 \pm 32.18$ & $<0.001$ \\
Surplus hemodynamic energy (\%) & $-63.79 \pm 22.03$ & $-75.37 \pm 8.21$ & $<0.001$ \\
\hline
\end{tabular}

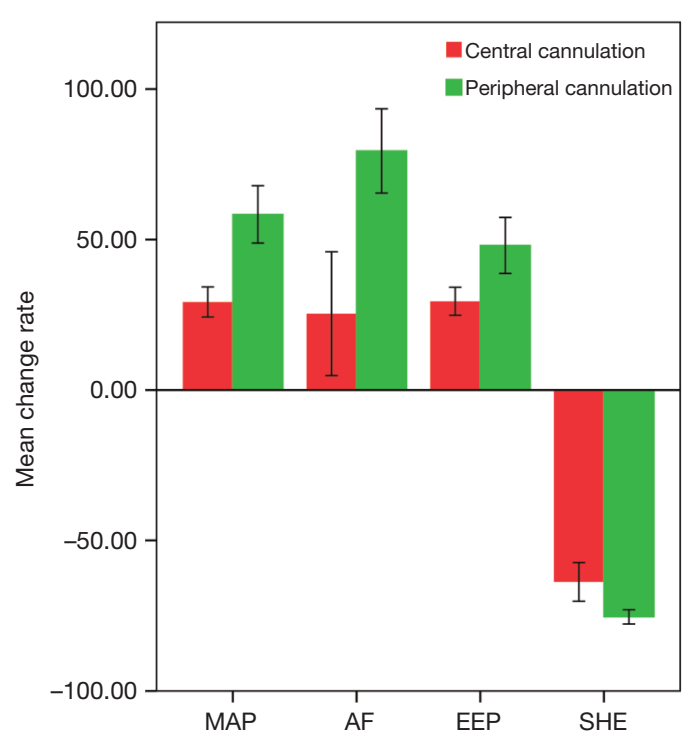

Figure 2 Comparison of rate of change in hemodynamic parameters, including mean arterial pressure (MAP), Arterial flow $(\mathrm{AF})$, energy equivalent pressure (EEP), and surplus hemodynamic energy (SHE), before and after initiation of extracorporeal membrane oxygenation using peripheral and central approaches (all $\mathrm{P}<0.001)$. Central cannulation—ascending aorta return cannular; Peripheral cannulation-femoral artery return cannular.

\section{Comparison of kidney injury biomarkers}

On inter-group analysis, change in BUN, creatinine, cystatin $\mathrm{C}$, and NGAL showed no significant differences between the 2 groups $(\mathrm{P}>0.05)$ (Table 3, Figure 3).

\section{Discussion}

Our results showed that both aECMO and fECMO showed increased MAP and AF compared with baseline values, as well as decreased SHE values. However, fECMO was associated with a significantly higher rate of change for $\mathrm{MAP}, \mathrm{AF}$, and EEP, and a decreased rate of change for SHE compared with aECMO. Therefore, our experiment demonstrated that fECMO had a higher total energy level and flow rate and decreased pulsatile energy compared with aECMO. There were no significant differences in BUN, creatinine, cystatin $\mathrm{C}$, and NGAL values associated with acute kidney injury (AKI) between the 2 groups during the 6-h ECMO time.

$\mathrm{AKI}$ is the most common complication during ECMO support, and is associated with reduced survival. The incidence of AKI following ECMO is 30-50\% (1,5). Most patients developed AKI required renal replacement therapy. 
Table 3 Comparison of BUN, creatinine, cystatin C, and NGAL according to time

\begin{tabular}{lccccc}
\hline Laboratory test & Group & Before ECMO & After ECMO & $3 \mathrm{~h}$ & $\begin{array}{c}\text { P value } \\
\text { (inter-group) }\end{array}$ \\
\hline $\mathrm{Hb}(\mathrm{g} / \mathrm{dL})$ & Central $(\mathrm{N}=6)$ & $8.8 \pm 0.6$ & $8.5 \pm 0.2$ & $8.3 \pm 0.8$ & $7.7 \pm 0.3$ \\
& Peripheral $(\mathrm{N}=6)$ & $9.1 \pm 0.9$ & $8.4 \pm 1.4$ & $8.9 \pm 1.3$ & $8.9 \pm 1.9$ \\
$\mathrm{BUN}(\mathrm{mg} / \mathrm{dL})$ & Central $(\mathrm{N}=6)$ & $5.8 \pm 0.8$ & $5.3 \pm 1.2$ & $5.9 \pm 1.0$ & $7.6 \pm 1.2$ \\
& Peripheral $(\mathrm{N}=6)$ & $7.9 \pm 2.4$ & $8.81 \pm 2.4$ & $9.1 \pm 2.5$ & $11.1 \pm 2.6$ \\
Creatinine $(\mathrm{mg} / \mathrm{dL})$ & Central $(\mathrm{N}=6)$ & $1.27 \pm 0.15$ & $1.16 \pm 0.21$ & $1.14 \pm 0.18$ & $1.11 \pm 0.18$ \\
& Peripheral $(\mathrm{N}=6)$ & $1.24 \pm 0.12$ & $1.23 \pm 0.21$ & $1.16 \pm 0.17$ & $1.08 \pm 0.14$ \\
Cystatin C $(\mathrm{mg} / \mathrm{L})$ & Central $(\mathrm{N}=6)$ & $2.39 \pm 2.5$ & $4.59 \pm 4.06$ & $3.60 \pm 3.21$ & $3.06 \pm 2.56$ \\
& Peripheral $(\mathrm{N}=6)$ & $2.26 \pm 1.77$ & $4.30 \pm 3.20$ & $3.50 \pm 2.94$ & $3.21 \pm 2.47$ \\
NGAL $(\mathrm{ng} / \mathrm{mL})$ & Central $(\mathrm{N}=6)$ & $1,167.2 \pm 761.5$ & $903.6 \pm 736.8$ & $671.9 \pm 253.6$ & $798.4 \pm 682.7$ \\
& Peripheral $(\mathrm{N}=6)$ & $1,514.2 \pm 762.4$ & $1,315.8 \pm 853.3$ & $1,110.3 \pm 543.24$ & $1,297.2 \pm 859.8$ \\
\hline
\end{tabular}

Data was presented as mean and standard deviation. $\mathrm{Hb}$, hemoglobin; BUN, blood urea nitrogen; NGAL, neutrophil gelatinase-associated lipocalin.
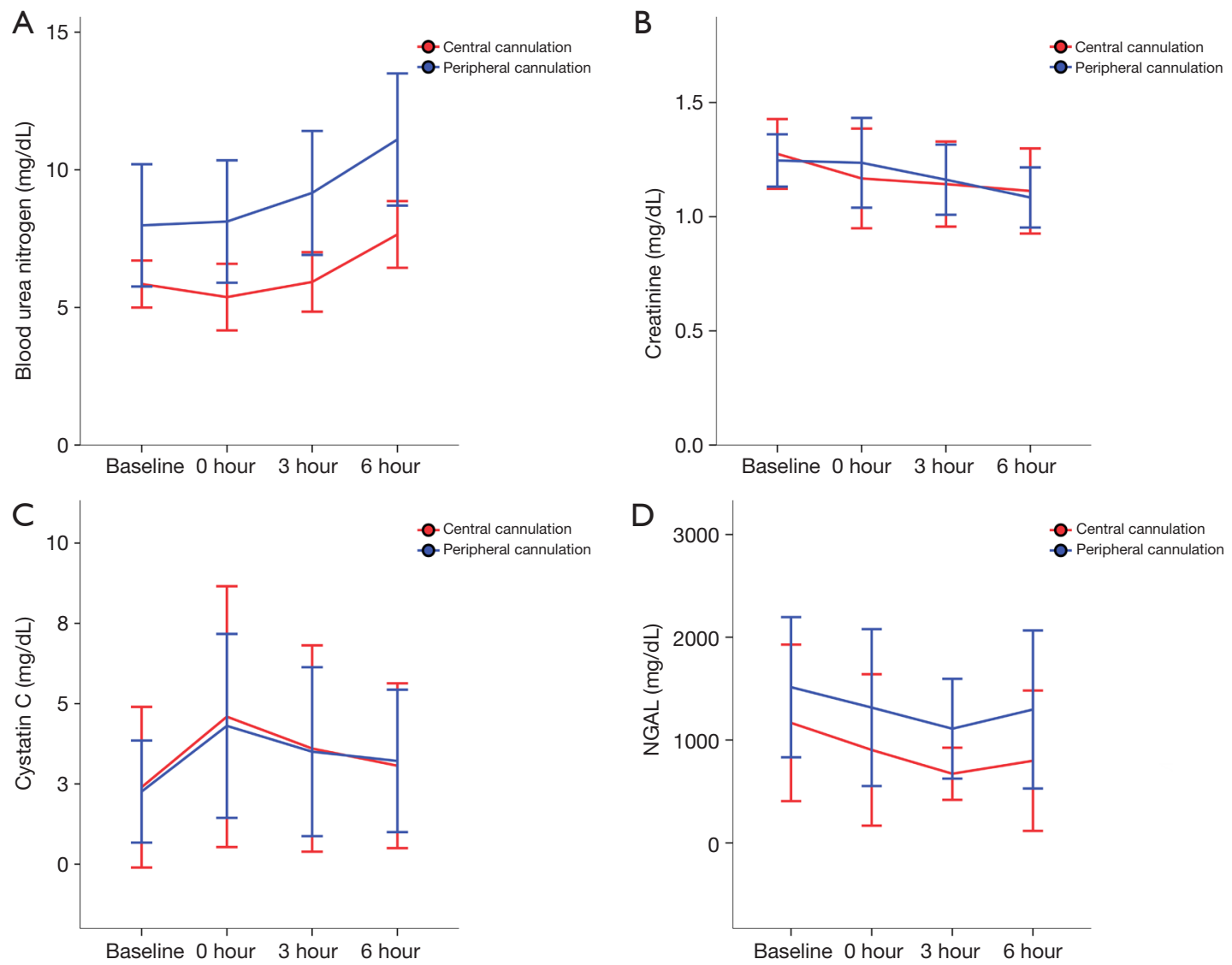

Figure 3 Comparison of biomarker levels, including blood urea nitrogen (BUN), creatinine, cystatin C, and neutrophil gelatinase-associated lipocalin (NGAL), using peripheral and central extracorporeal membrane modes according to time interval (all $\mathrm{P}$ value were no significant). Central cannulation—ascending aorta return cannular; Peripheral cannulation—femoral artery return cannular. 
The occurrence of AKI varies with risk factors or ECMO method (5). Factors that influence AKI include low cardiac output, nephrotoxic agents, infection, and inflammatory reactions induced by the ECMO device. Hemodynamic instability plays an important role and can result in irreversible loss of kidney function and worsening of preexisting kidney dysfunction. Therefore, the preservation or restoration of adequate blood flow and pressure may reduce the risk of AKI.

The role of return cannula (arterial cannula) position in organ preservation and complications is unclear. In aECMO, placing the arterial cannula near the heart offers effective perfusion for the heart and brain and provides antegrade flow, as in normal circulation. In contrast, in fECMO, placing the arterial cannula distal to the heart (mostly in the femoral artery) simplifies cannulation but is disadvantageous in terms of distance from major organs; moreover, the retrograde flow is opposite that of natural circulation. Some clinical studies regarding cannular position showed equivocal results between aECMO and $\mathrm{FECMO}$ in terms of complications and survival rate $(10,11,13)$. An experimental study attempted to evaluate hemodynamic flow change according to return cannular position by computational fluid dynamics (14). However, hemodynamics in the kidney in vitro was not well evaluated according to cannular position. We addressed the effect of energy delivery according to different cannula positions by measuring MAP, EEP, and SHE. This study showed that when cardiac output was replaced with ECMO-generated flow, the rate of change in total energy at the renal artery was higher in subjects receiving fECMO than in those with aECMO. This results might be derived from fECMO placement of arterial return closer to the renal artery, with fewer branches from the upper aorta than with aECMO.

Pulsatility of blood flow is also important in efficient energy delivery. Some studies showed energy loss with non-pulsatile flow compared with that in pulsatile flow. According to those reports, non-pulsatile flow delivered less energy to target organs than pulsatile flow (18-21). Centrifugal mode ECMO generates non-pulsatile flow through the return cannula. Non-pulsatile flow generated by ECMO merges with the circulating pulsatile flow from the heart. Our study showed that pulsatility energy represented as SHE was decreased in both aECMO and fECMO. However, reduced pulsatility was more significant in swine with $\mathrm{EECMO}$ than in those with aECMO. This difference may affect recovery from impaired renal function during ongoing kidney injury.
Biomarker testing is highly sensitive for detection of tissue injury or functional renal impairment $(22,23)$. In our study, ECMO with preserved energy did not show a significant change in several biomarkers. When the kidneys are damaged, the concentration of NGAL and cystatin C increases in urine and blood within a few hours. While previous published studies were conducted using ischemicreperfusion conditions, our study was performed with preserved kidney function. Therefore, in our experimental setting, kidney function was not influenced by optimized ECMO support.

\section{Limitation}

A limitation of this study is the small number of cases due to in vivo test. To evaluate the impact of hemodynamics on kidney injury, an injured kidney model may be better than our normal kidney model. However, our study model evaluated hemodynamic parameters without confounding factors. A major limitation of this study was relative shorter operation time of ECMO than that of a usual clinical setting. However, it is enough to collect hemodynamic change according to control factors and longer time may increase confounding factors such as bleeding and infection. Biomarkers used in this study have short onset times less than 2-3 hours. Therefore, kidney injury can be reflected in the laboratory test.

\section{Conclusions}

ECMO with a peripheral arterial cannula (femoral artery) showed a higher energy level (EEP) at the renal artery than with use of a central arterial cannula (ascending aorta). However, pulsatility loss was greater in fECMO than in aECMO.

\section{Acknowledgments}

This work was supported by a Korea University Grant.

\section{Footnote}

Conflicts of Interest: The authors have no conflicts of interest to declare.

Ethical Statement: The authors are accountable for all aspects of the work in ensuring that questions related to the accuracy or integrity of any part of the work are 
appropriately investigated and resolved. This study was approved by Korea University Institutional Animal Care and Use Committee. All animals were treated according to the Guide for the Care and Use of Laboratory Animals issued by the Korea University School of Medicine.

\section{References}

1. Cheng R, Hachamovitch R, Kittleson M, et al. Complications of extracorporeal membrane oxygenation for treatment of cardiogenic shock and cardiac arrest: a meta-analysis of 1,866 adult patients. Ann Thorac Surg 2014;97:610-6.

2. Aso S, Matsui H, Fushimi K, et al. In-hospital mortality and successful weaning from venoarterial extracorporeal membrane oxygenation: analysis of 5,263 patients using a national inpatient database in Japan. Critical Care 2016;20:80.

3. Sultan I, Habertheuer A, Wallen T, et al. The role of extracorporeal membrane oxygenator therapy in the setting of Type A aortic dissection. J Card Surg 2017;32:822-5.

4. Husain-Syed F, Ricci Z, Brodie D, et al. Extracorporeal organ support (ECOS) in critical illness and acute kidney injury: from native to artificial organ crosstalk. Intensive Care Med 2018;44:1447-59.

5. Chen YC, Tsai FC, Fang JT, et al. Acute kidney injury in adults receiving extracorporeal membrane oxygenation. J Formos Med Assoc 2014;113:778-85.

6. Kudaka M, Kuniyoshi Y, Miyagi K, et al. Experimental study on changes in energy metabolism and urine outflow with nonpulsatile low blood-flow perfusion in the canine kidney. Jpn J Thorac Cardiovasc Surg 2003;51:225-31.

7. Arnaoutakis GJ, Vallabhajosyula P, Bavaria JE, et al. The Impact of Deep Versus Moderate Hypothermia on Postoperative Kidney Function After Elective Aortic Hemiarch Repair. Ann Thorac Surg 2016;102:1313-21.

8. Kumar AB,Suneja M. Cardiopulmonary Bypass-associated Acute Kidney Injury. Anesthesiology 2011;114:964-70.

9. Murphy GS, Hessel EA, Groom RC. Optimal Perfusion During Cardiopulmonary Bypass: An Evidence-Based Approach. Anesth Analg 2009;108:1394-417.

10. Saeed D, Stosik H, Islamovic M, et al. FemoroFemoral Versus Atrio-Aortic Extracorporeal Membrane Oxygenation: Selecting the Ideal Cannulation Technique. Artif Organs 2014;38:549-55.

11. Benedetto U, Raja SG, Amrani M, et al. The impact of arterial cannulation strategy on operative outcomes in aortic surgery: Evidence from a comprehensive metaanalysis of comparative studies on 4476 patients. J Thorac Cardiovasc Surg 2014;148:2936-43.e1-4.

12. Kamimura T, Sakamoto H, Misumi K. Regional blood flow distribution from the proximal arterial cannula during veno-arterial extracorporeal membrane oxygenation in neonatal dog. J Vet Med Sci 1999;61:311-5.

13. Loforte A, Marinelli G, Musumeci F, et al. Extracorporeal Membrane Oxygenation Support in Refractory Cardiogenic Shock: Treatment Strategies and Analysis of Risk Factors. Artif Organs 2014;38:E129-41.

14. Gu KY, Zhang Y, Gao B, et al. Hemodynamic Differences Between Central ECMO and Peripheral ECMO: A Primary CFD Study. Med Sci Monit 2016;22:717-26.

15. Son KH, Ahn CB, Lee SH, et al. Measurement of Hemodynamic Energy at Different Vessels in an Adult Swine Model. ASAIO J 2010;56:397-402.

16. Undar A, Rosenberg G, Myers JL. Major factors in the controversy of pulsatile versus nonpulsatile flow during acute and chronic cardiac support. ASAIO J 2005;51:173-5.

17. Undar A. Myths and truths of Pulsatile and nonpulsatile perfusion during acute and chronic cardiac support. Artif Organs 2004;28:439-43.

18. Voicu S, Sideris G, Dillinger JG, et al. Synchronized Pulsatile Flow With Low Systolic Output From VenoArterial Extracorporeal Membrane Oxygenation Improves Myocardial Recovery After Experimental Cardiac Arrest in Pigs. Artif Organs 2018;42:597-604.

19. Voss B, Krane M, Jung C, et al. Cardiopulmonary bypass with physiological flow and pressure curves: pulse is unnecessary! Eur J Cardiothorac Surg 2010;37:223-32.

20. Kim HK, Son HS, Fang YH, et al. The effects of pulsatile flow upon renal tissue perfusion during cardiopulmonary bypass: A comparative study of pulsatile and nonpulsatile flow. ASAIO J 2005;51:30-6.

21. Undar A, Masai T, Yang SQ, et al. Effects of perfusion mode on regional and global organ blood flow in a neonatal piglet model. Ann Thorac Surg 1999;68:1336-42; discussion 42-3.

22. Coca SG, Yalavarthy R, Concato J, et al. Biomarkers for the diagnosis and risk stratification of acute kidney injury: A systematic review. Kidney Int 2008;73:1008-16.

23. Ricci Z, Morelli S, Favia I, et al. Neutrophil gelatinaseassociated lipocalin levels during extracorporeal membrane oxygenation in critically ill children with congenital heart disease: Preliminary experience. Pediatr Crit Care Med 2012;13:E51-4.

Cite this article as: Kim HJ, Jeong SC, Jung JS, Kim IS, Lim $\mathrm{CH}$, Son HS. Renal hemodynamics by return cannular position of extracorporeal membrane oxygenation in swine. J Thorac Dis 2019;11(10):4211-4217. doi: 10.21037/jtd.2019.09.73 